\title{
EVALUATION OF NEUTROPHILS IMMUNOPHENOTYPE IN THE MICROENVIRONMENT OF MALIGNANT PLEURAL EFFUSIONS
}

\author{
Mariusz Kaczmarek ${ }^{1 *}$, Joanna Maciejewska ${ }^{1}$, Łukasz Spychalski ${ }^{2}$, Magdalena Socha-Kozłowska ${ }^{2}$, \\ Agata Nowicka ${ }^{3}$, Jan Sikora ${ }^{1}$
}

\begin{abstract}
The lung cancer is often associated with the development of pleural effusion. Neutrophils are the most numerous population of immune system cells which are an essential component of tumor leukocyte infiltration. These cells are engaged in the development and maintenance of the inflammation. It is indicated that neutrophils support the development of cancer. The aim of the study was the evaluation of neutrophils, regarding their presence and activity in pleural effusions. This was achieved by assessing of molecular structures, which are used by neutrophils in chemotaxis and phagocytosis.

60 pleural effusions and 34 peripheral blood samples received from patients and 15 peripheral blood samples from the control group were analyzed. Expression of CD11a, CD11b, CD11c, CD18 and CD62L molecules with use flow cytometry was evaluated. The concentration of the neutrophil elastase in pleural effusions were measured with use ELISA test.

The number of neutrophils in the peripheral blood of patients with pleural effusion was lower than that observed in the control group. Neutrophils present in pleural effusions were characterized by an increased ability to chemotaxis and secrete significant amounts of neutrophil elastase.

Neutrophils recruited into the pleura during the formation of the effusion are an essential element of the developing inflammatory reaction in this environment. The presence of neutrophils in pleural effusion may promote its further formation and support the development of cancer.
\end{abstract}

Running title: Neutrophils in malignant pleural effusions

Keywords: neutrophils, pleural effusion, lung cancer, integrins

\footnotetext{
${ }^{1}$ Department of Immunology, Chair of Clinical Immunology, Poznan University of Medical Sciences, Poznan, Poland

${ }^{2}$ Wielkopolskie Centre of Pulmonology and Thoracic Surgery, Poznan, Poland

${ }^{3}$ Department of Pulmonology, Allergology and Respiratory Oncology, Poznan University of Medical Sciences, Poznan, Poland

*Correspondence: markacz@ump.edu.pl

Full list of author information is available at the end of article
} 


\section{Introduction}

In the lung cancer, a frequent complication is an accumulation in the pleural cavity of an excessive amount of pleural fluid. Pleural effusion arises during the development of primary pleural cancer, the mesothelioma, but more frequently it is the result of the metastasis of cancer cells of the non-small cell lung cancer (NSCLC). Malignant effusions are also associated with metastases of breast cancer, ovarian cancer, and lymphomas [1]. Presence of effusion in a pleural cavity is a very unfavorable prognostic factor. The average survival time for a patient with effusion is about six months, for lung cancer or lymphoma, and 48 months for breast cancer [2]. Malignant effusion creates an environment promoting the survival and spread of cancer cells.

Under physiological conditions, the pleural fluid contains mesothelial cells, macrophages, lymphocytes and sometimes neutrophils [3]. In pathological conditions, the amount of monocytes, neutrophils, and eosinophils significantly increases [4]. Neutrophils are the largest group of peripheral blood leukocytes. These cells are a crucial element of the innate immune response and constitute the first line of defense against penetrating pathogens. These granulocytes are highly mobile and are the first cells to appear in the place of inflammation. Neutrophils circulating in the peripheral blood overcome the barrier that is the endothelium of the blood vessels to reach the place of inflammation. The process of diapedesis is directed and strictly controlled at the molecular level. In the first stage of migration through the vascular endothelium, neutrophils use L-selectin (CD62L) and type-1 ligand for P-selectin (PSGL-1, CD162) which bind to $\mathrm{P}$ (CD62P) and E (CD62E) selectins presented on the surface of epithelial cells [5]. Transmigration of the endothelial barrier by granulocytes and their infiltration into the extravascular space provide integrins, LFA-1 (CD11a/CD18; $\alpha_{\mathrm{L}} \beta_{2}$-complex) and Mac-1 (CD11b/CD18; CR3; $\alpha_{\mathrm{M}} \beta_{2}$-complex). The ligands for these integrins are intercellular adhesion molecules ICAM-1 and ICAM-2 presented on the surface of vascular endothelial cells [6]. Neutrophil recruitment appears in response to chemotactic factors associated with infection as well as processes that require the activation of a pool of neutrophils present in the bone marrow [7]. Factors stimulating the neutrophil chemotaxis are interleukin 8 (IL-8), interleukin 1 (IL-1), complement components C3a and C5a, $\mathrm{N}$-Formylmethionine-leucyl-phenylalanine (fMLP), also leukotriene $\mathrm{B}_{4}\left(\mathrm{LTB}_{4}\right)$ [8].

Neutrophils belong to professional phagocytic cells. They eliminate pathogens by the phagocytosis, in the dependent and independent way from opsonins. For this purpose, these cells use a broad repertoire of phagocytic receptors, including receptors for immunoglobulins - Fc $\gamma$ RI (CD64), Fc $\gamma$ RIIa (CD32), FcyIIIb (CD16), and Fc $\gamma$ RIV, as well as for complement components - CR1, CR3, CR4 [9]. Integrins CD11b/CD18 (CR3) and CD11c/CD18 (LeuM5, CR4, $\alpha_{x 2}$-complex) play a dominant role in the phagocytosis of bacteria opsonized by $\mathrm{iC} 3 \mathrm{~b}$ and $\mathrm{C} 4 \mathrm{~b}$ [10]. The pathogen's death within phagolysosome is the result of the activation of cytotoxic mechanisms, dependent and independent of oxygen. The most effective mechanism of intracellular killing is the oxidative burst [11]. The neutrophil elastase (NE) accumulated in azurophilic granules belongs to the family of serine proteases with high bactericidal activity [12]. The spectrum of substrates of neutrophil elastase are proteins that are part of the extracellular matrix (ECM), such as elastin, collagen type IV, fibronectin and proteoglycans [13-15]. Neutrophil elastase is used in diapedesis, destroys bacterial and viral proteins, and together with myeloperoxidase participates in the formation by neutrophils of neutrophil extracellular traps (NET), which support the process of killing bacteria [16].

The present study aimed to determine the presence and activity of neutrophils in pleural effusions associated with non-small cell lung cancer. This was achieved by determining the percentages of neutrophils in pleural effusions, and peripheral blood of patients with NSCLC accompanied with pleural effusion. Neutrophils were evaluated for the expression of CD62L selectin, and LFA-1, Mac-1, and LeuM5 integrins, also the concentration of neutrophil elastase was measured in the studied pleural effusions.

\section{Material and methods \\ Pleural effusions and peripheral blood}

The studied material included pleural effusions obtained via thoracocentesis from 60 patients (37 malignant and 23 non-malignant) hospitalized in Wielkopolskie Centre of Pulmonology and Thoracic Surgery in Poznan and in Department of Pulmonology, Allergology and Respiratory Oncology of Poznan University of Medical Sciences. From 34 patients, approximately $3 \mathrm{ml}$ of peripheral blood was collected into tubes with the anticoagulant, K2-EDTA (Potassium-EDTA, Sarstedt-Monovette ${ }^{\circledR}$ ). The control consisted of 15 peripheral blood samples from participants without cancer disease. The biological material used in the research was collected in accordance with the decision of the Poznan University of Medical Sciences Bioethics Committee No. 59/14 of January 16, 2014.

Pleural effusions were filtered through three-layered sterile gauze and then centrifuged at $300 \mathrm{~g}$ in $50 \mathrm{ml}$ tubes (BD Falcon ${ }^{\mathrm{TM}}$ ) for $10 \mathrm{~min}$ at room temperature. Supernatants were frozen and stored at $70^{\circ} \mathrm{C}$ until ELISA tests were performed. Cell pellets obtained from pleural effusions and samples of peripheral blood were directly subjected to cytometric analysis. For the cytological assessment from the part of cellular pellets, smears were made, and then stained with hematoxylin and eosin $(\mathrm{H}+\mathrm{E})$. 


\section{Flow cytometric evaluation}

The immunophenotypic assessment was performed on fresh, non-fixed cells from pleural effusions and peripheral blood samples. The studied material was added to the polypropylene tubes, where was mixed with the monoclonal antibodies (Tab. 1). Samples were incubated for $15 \mathrm{~min}$ at room temperature, protected from light. Negative controls were cells without added detecting antibodies. Then erythrocytes were lysed, and samples were washed twice by centrifugation at 1500 rpm for $5 \mathrm{~min}$ at $4 \mathrm{C}$. The material prepared in this manner was subjected to acquisition using FACS Canto flow cytometer (Becton Dickinson), and the obtained results were analyzed using the FASC Diva software (Becton Dickinson).

\section{Evaluation of neutrophil elastase concentration}

Centrifuged and frozen supernatants from pleural effusions were thawed and brought to room temperature. Determination of NE concentration was performed using a commercially available sandwich ELISA (BioVendor) test by the attached manufacturer's protocol. Appropriate standards were used for the quantitative assessment. The assay was performed based of on the controls with low and high concentrations of the tested protein (LOW and HIGH). The intensity of the color product of horseradish peroxidase reaction was read with a microtiter reader at the wavelength $\lambda=450 \mathrm{~nm}$.

\section{Statistical analysis}

For statistical analysis non-parametric tests Mann-Whitney U and Kruskal-Wallis were used. All tests were carried out in a confidence interval equal to $=0.95$ at the significance level $p=0.05$. For determination of a correlation between the percentage of neutrophils in the examined effusions and the concentration of NE the Spearman rank correlation test was used. Calculations were conducted with GraphPad Prism version 4.03 (GraphPad Software) software.

\section{Ethical approval:}

The research related to human use has been complied with all the relevant national regulations, institutional policies and in accordance the tenets of the Helsinki Declaration, and has been approved by the authors' institutional review board or equivalent commitee. Poznan University of Medical Sciences Bioethics Committee approval no. 59/14 of January 16, 2014.

\section{Results}

Immunophenotype assessment of neutrophils

Neutrophils in the test samples were determined based on the presented immunophenotype: $\mathrm{FSC}^{\text {high }}$ / $\mathrm{SSC}^{\text {high }} / \mathrm{CD} 45^{+} / \mathrm{CD} 14^{-} / \mathrm{CD} 15^{+} / \mathrm{HLA}^{-D R}{ }^{-}$(Fig. 1a). The cells selected in this way were further evaluated for the expression of CD11a, CD11b, CD11c, CD18 and CD62L antigens. The Mean Fluorescence Intensity (MFI) values of the receptors were determined during the analysis. The observed MFI values for a particular fluorochrome corresponded to the density of the expressed molecules (Fig. 1b).

The highest percentage of neutrophils was found in the peripheral blood of patients with pleural effusions. The observed amount slightly differed in comparison to the amount of neutrophils in peripheral blood control samples. The Kruskal-Wallis test showed that the neutrophil percentage present in pleural effusions was statistically significantly lower than in the peripheral blood of patients at $p=0.000003$ and in the peripheral blood of the control group at $p=0.000002$ (Fig. 2).

\section{Expression of CD11a/CD11b/CD11c/CD18 molecules}

The value of the mean fluorescence intensity of the CD11a molecule was the highest on the surface of neutrophils from pleural effusions (Fig. 3a). The Kruskal-Wallis test showed that the difference observed between MFIs for the CD11a molecule presented on the surface of neutrophils from pleural effusion and neutrophils from the peripheral blood of patients is statistically significant at $\mathrm{p}=0.034747$.

TABLE 1 Antibodies used for evaluation of neutrophils immunophenotype

\begin{tabular}{|c|c|c|c|c|}
\hline Antibodies & Fluorochrome & Idiotype & Clone & Source \\
\hline anti-CD45 & APC-Сy7 & $\operatorname{IgG}_{1}, \kappa$ & 2D1 & BD Biosciences \\
\hline anti-CD14 & $\mathrm{PE}$ & $\operatorname{Ig} G_{1}, \kappa$ & rmC5-3 & BD Biosciences \\
\hline anti-CD15 & APC & $\operatorname{IgM}, \kappa$ & HI98 & BD Biosciences \\
\hline anti-HLA-DR & PerCP & $\operatorname{IgG}_{2}, \kappa$ & L243 & BD Biosciences \\
\hline anti-CD11a & FITC & $\operatorname{Ig} G_{2 a^{\prime}} \kappa$ & G-25.2 & BD Biosciences \\
\hline anti-CD11b & APC & $\operatorname{IgG}_{2 \mathrm{a}^{\prime}} \kappa$ & D12 & BD Biosciences \\
\hline anti-CD11c & PE & $\operatorname{IgG}_{1}, \lambda 2$ & HL3 & BD Biosciences \\
\hline anti-CD18 & FITC & $\operatorname{IgG}_{1}, \kappa$ & L130 & BD Biosciences \\
\hline anti-CD62L & $\mathrm{PE}$ & $\operatorname{IgG}_{2 a}, \kappa$ & SK11 & BD Biosciences \\
\hline
\end{tabular}


The highest MFI value of the CD11b molecule was observed on the surface of neutrophils derived from pleural effusions (Fig. 3b). The Kruskal-Wallis test showed that the observed difference between MFI for CD11b on neutrophil from pleural effusions and MFI for CD11b on neutrophils in peripheral blood of pa- tients at $\mathrm{p}=0.000034$ and peripheral blood of the control group at $p=0.000241$ was statistically significant.

The MFI of the CD11c molecule on the neutrophil surface in all tested materials showed no differences at the statistically significant, at level $p=0.2764$ (Fig. 3c).

\section{a. scattergrams}

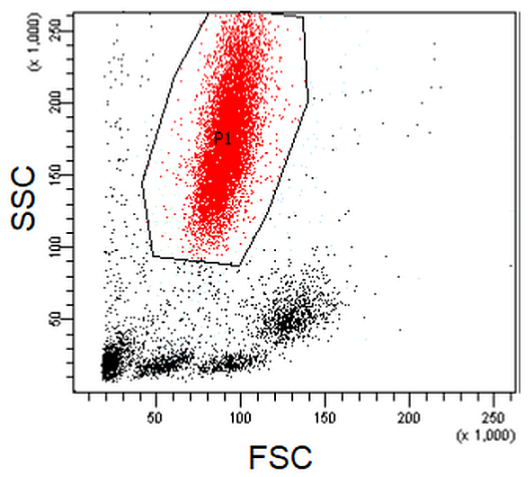

b. histograms

CD11b

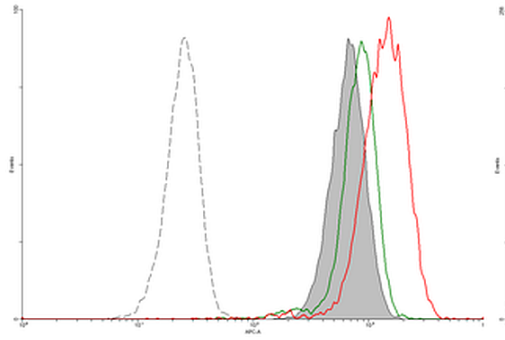

blood - control
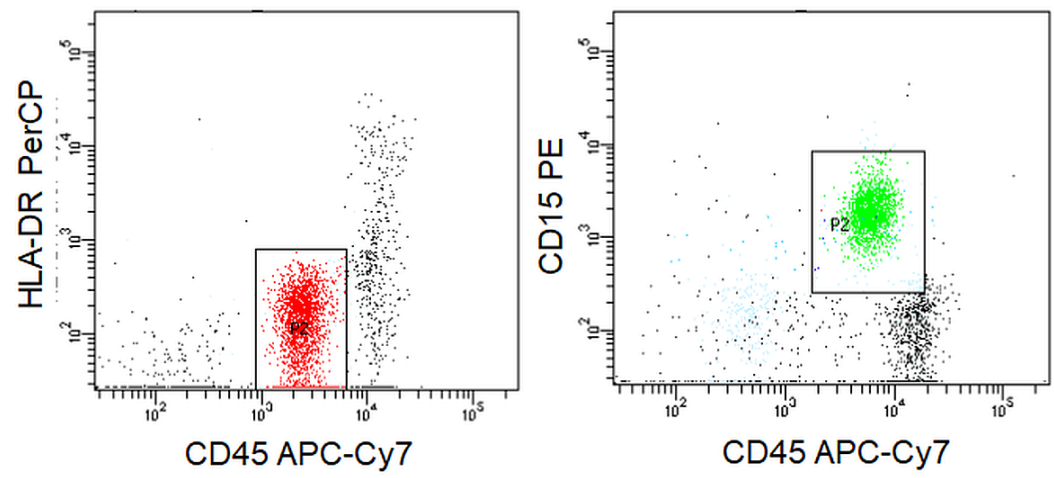

CD18

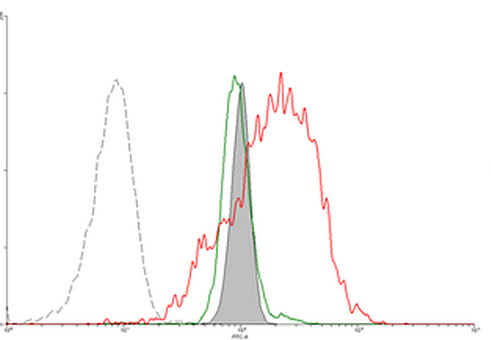

blood - patients
CD62L

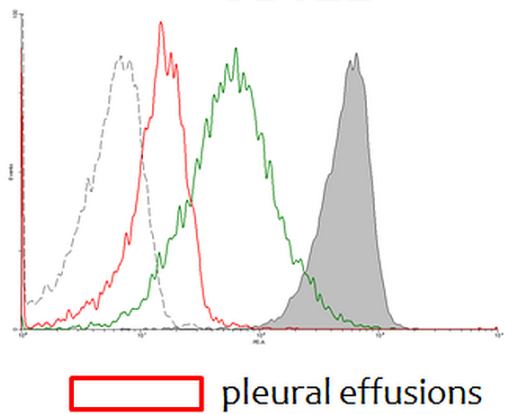

Figure 1 Flow cytometric scattergrams presenting an example of neutrophil gating (a.); histograms presenting mean fluorescent intensity of CD11b, CD18, and CD62L molecules measured on the surface of neutrophils in peripheral blood and pleural effusions of patients, as well as in the peripheral blood of control group

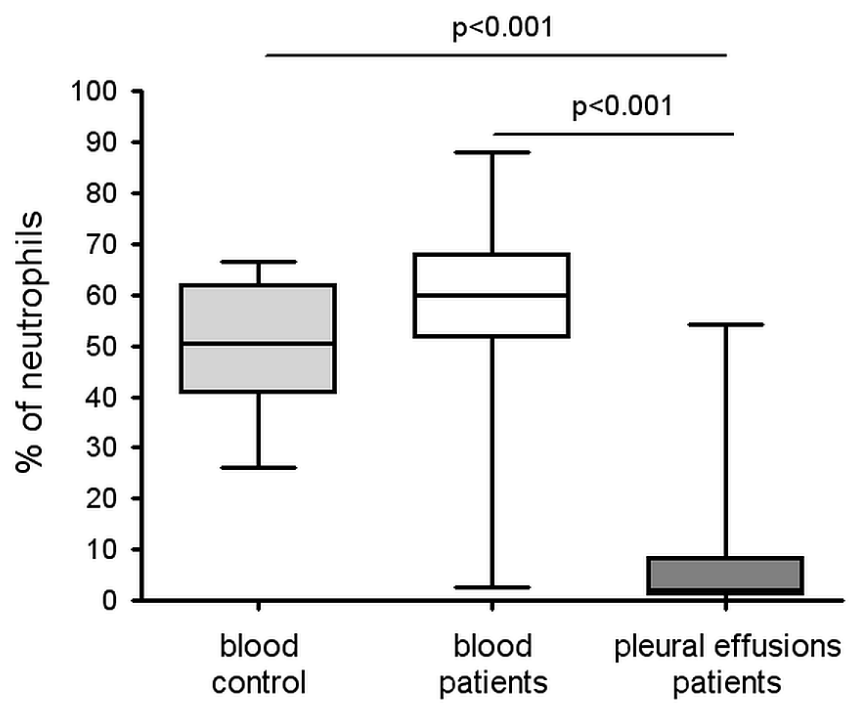

Figure 2 Percentage of neutrophils in studied materials. Observed differences are statistically important at presented p-values 
The MFI value for the CD18 molecule observed on the surface of neutrophils from pleural effusions was higher than the MFI values found for the CD18 molecules on the neutrophil surface from the peripheral blood of the control group and peripheral blood of patients with pleural effusions (Fig. 4). The Kruskal-Wallis test showed that the observed differences were statistically significant, at $p=0.003583$ for peripheral blood neutrophils from the control group, and at $p=0.000001$ for neutrophils in the peripheral blood of patients with pleural effusions.

\section{Expression of the CD62L molecule}

Evaluation of expression of the CD62L molecule showed that the MFI observed on the surface of neutrophils derived from pleural effusions was lower in relation to neutrophils from the peripheral blood of patients with pleural effusion and peripheral blood from the control group (Fig. 5). The Kruskal-Wallis test showed that the difference between the MFI levels of CD62L molecules presented on the surface of neutrophils from peripheral blood in the control group and from pleural effusions was statistically significant at $\mathrm{p}=0.001834$. The difference between the MFI levels of CD62L molecules presented on the surface of neutrophil in the peripheral blood of patients developing pleural effusions and on the surface of neutrophils in pleural effusions was also statistically significant at $\mathrm{p}=0.001834$.

\section{Concentration of neutrophil elastase (NE) in pleural effusions}

Mean concentration of neutrophil elastase in non-malignant pleural effusions $(7.85 \mu \mathrm{g} / 100 \mathrm{mg}$ of the total protein) was higher than the mean concentration in pleural effusions of malignant etiology (7.40 $\mu \mathrm{g} / 100 \mathrm{mg}$ of the total protein) (Fig. 6). The U Mann-Whitney's test showed that the difference observed between concentration levels of the neutrophil elastase in pleural effusions with different etiology was not statistically significant, at p-value 0.630669 . a.

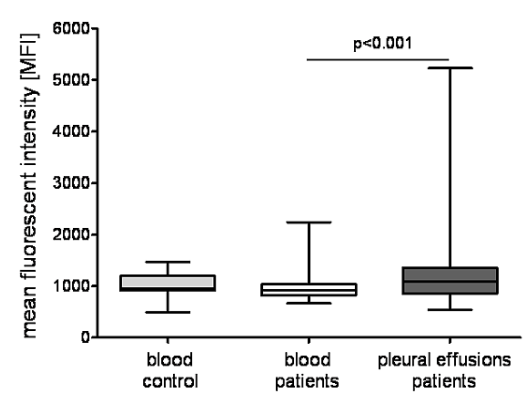

b.

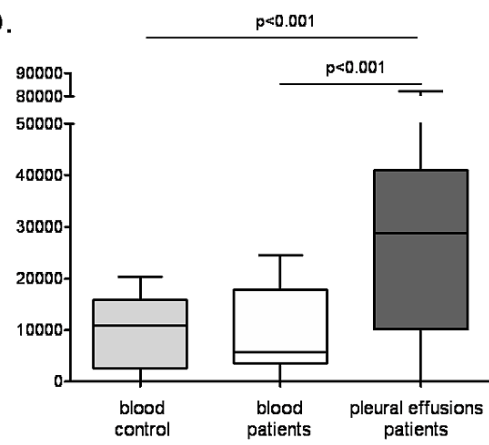

C.

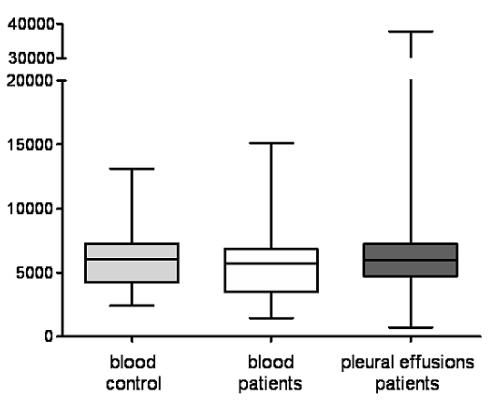

Figure 3 Level of expression of CD11a (a.); CD11b (b.), and CD11c (c.) molecules on the studied neutrophils described as a mean fluorescent intensity value [MFI]

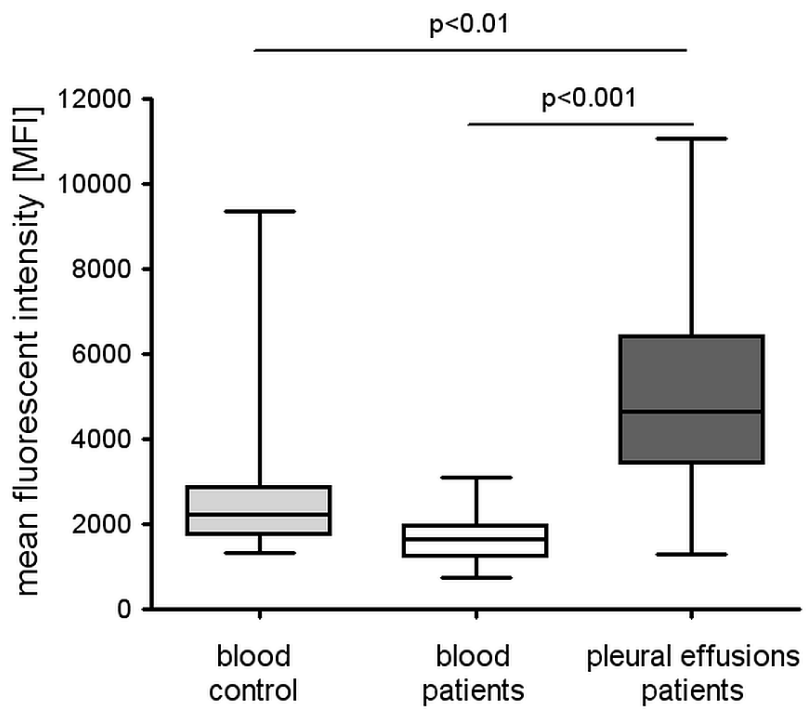

Figure 4 Level of expression of CD18 molecules on the studied neutrophils described as a mean fluorescent intensity value $[\mathrm{MFI}]$ 


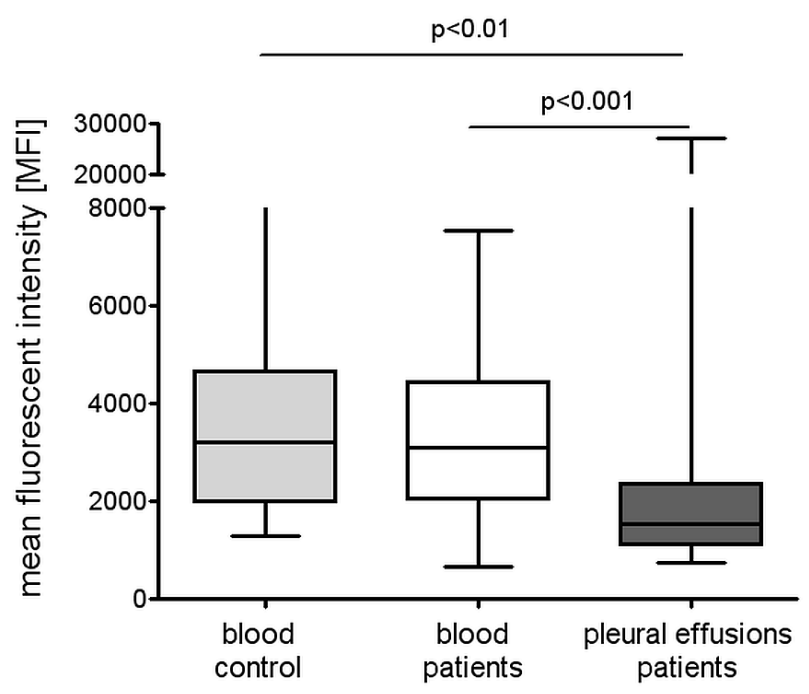

Figure 5 Level of expression of CD62L molecules on the studied neutrophils described as a mean fluorescent intensity value $[\mathrm{MFI}]$

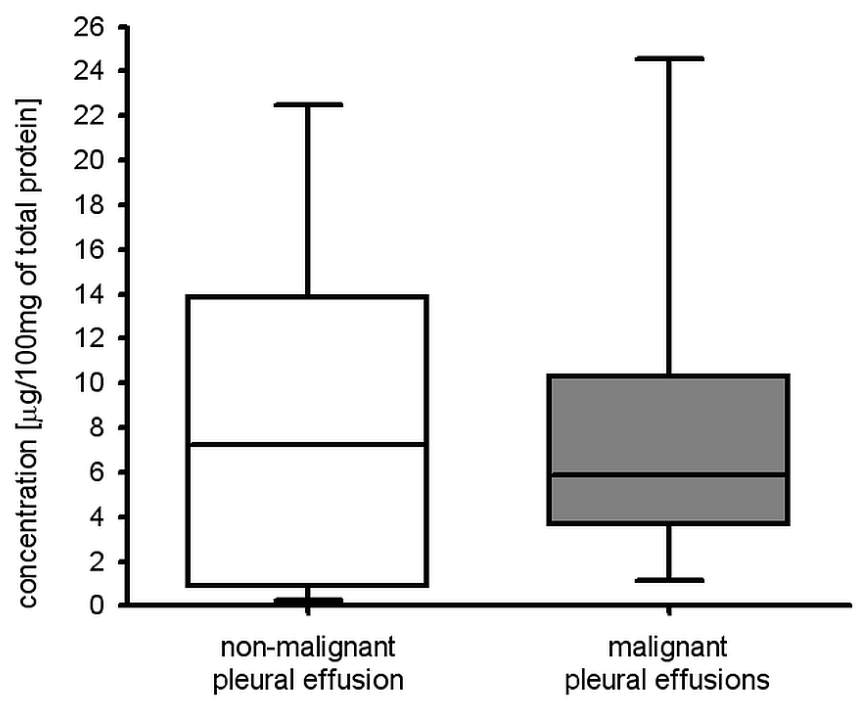

Figure 6 Comparison of values of neutrophil elastase (NE) concentrations determined in malignant and non-malignant pleural effusions. The concentration was measured against to the $100 \mathrm{mg} /$ of the total protein concentration present in the studied pleural effusions

\section{Discussion}

Neutrophils present in the tumor microenvironment are named tumor-associated neutrophils (TAN). These cells under the influence of the factors synthesized and secreted by the tumor, or other cells present in the tumor environment, change their functional properties. Suppression mediated by the tumor causes that neutrophils reprogram their natural anticancer activity and support the cancer development and the pro-tumor activity of various components of the immune system. Through promotion angiogenesis, neutrophils support the growth, development and even the spreading of cancer. It was found that neutrophil-to-lymphocyte ratio (NLR) in the peripheral blood of patients with cancer can be used as a prognostic marker. In NSCLC patients, the high value of the
NLR parameter is an independent unfavorable prognostic factor $[17,18]$. Also, it has been observed that the number of neutrophils in the peripheral blood increases simultaneously with the development of cancer [19-21]. The large number of neutrophils infiltrating the tumor in the course of NSCLC is associated with the more advanced state of disease and poor prognosis [22]. The main chemotactic factor recruiting neutrophils to the infiltrate of the tumor is interleukin 8 (IL-8). Compared to healthy individuals, in patients with cancer IL-8 in peripheral blood demonstrated elevated levels [23]. The best-known mechanism for supporting the development of cancer by neutrophils is their effect on the development of angiogenesis. The crucial role of TAN in this process is the release of vascular endothelial growth factor (VEGF), as well as 
matrix metallopeptidase 9 (MMP-9), which contribute to the development of new blood vessels within the cancer microenvironment $[24,25]$. We have found a higher percentage of neutrophils in the peripheral blood of lung cancer patients with pleural effusion as compared to the peripheral blood of the control group. Even though the rate of neutrophils in pleural effusions was lower than in peripheral blood, the appearance of pleural effusion is always a more severe condition for the patient. The presence of malignant pleural effusion is associated with its systemic impact negatively affecting the quality of life of the patient, as well as enhancing the development of cancer.

Demonstrated by us changes in the expression of the L-selectin on the surface of neutrophils present in pleural effusion indicate increased mobility of these cells. This effect can be associated with the recruitment of neutrophils by pleural macrophages [26]. The level of expression of L-selectin on neutrophils present in pleural effusions and peripheral blood of patients developing pleural effusions was lower than on neutrophils in the peripheral blood of the control group. The observed potentially increased chemotactic activity of neutrophils in patients with pleural effusion is also confirmed by the increased expression both of LFA-1 and Mac-1 integrins In the presented studies, we showed an increased level of expression of CD11a and CD18 molecules (LFA-1-forming subunits) on neutrophils in pleural effusions and CD11b and CD18 (Mac-1-forming subunits) on neutrophils in pleural effusions and peripheral blood of patients developing pleural effusions. The increased expression of the Mac- 1 complex may also indicate the readiness of the studied neutrophils to the phagocytic activity in the pleural effusion microenvironment. However, this part of neutrophil activity would require additional confirmation by functional tests. Spicer JD and co-workers showed that neutrophils through Mac-1 integrin support the process of metastasis in the development of liver cancer in mice [27]. In our studies, we observed no statistically significant difference in the expression of CD11c antigen on neutrophils in the pleural effusion and the blood of the control group. This indicates that the CD11c subunit is not sensitive to factors present in the pleural effusions and is stable at the established level. It also may because suppressor factors presented in pleural effusion keep the expression of the CD11c molecule (LeuM5- forming subunit) on the not activated level and block the phagocytic activity of neutrophils. Changes in the level of the expression of functional receptors occurring on the surface of neutrophils in the pleural effusion indicate that this environment significantly modulates the conditions of immune cells' functioning. A mixture of different types of cells present in a molecular cocktail containing proinflammatory, suppressive and tumor-promoting factors may more holistically affect the organism, and not only locally at the site of the developing tumor. The presence of TAN combined with regulatory $\mathrm{T}$ cells (Treg), tumor-associated macrophages (TAM) and myeloid-derived suppressor cells (MDSC) in the cancer microenvironment can significantly support the development and spread of tumor and effectively inhibit the antitumor activity of immunocompetent cells [28].

The elastase secreted by neutrophils may be involved in the neoplastic process. Its pro-malignant activity is associated with proteolytic properties, which includes proteins of the extracellular matrix. The degradation of ECM-forming proteins promotes the escape of cells from the primary tumor, which supports the process of the formation of metastasis [29]. Houghton AM et al. showed that NE stimulates the growth of cancer cells by activating the signaling pathway associated with phosphatidylinositol kinase (PI3K). After penetration into the tumor cell

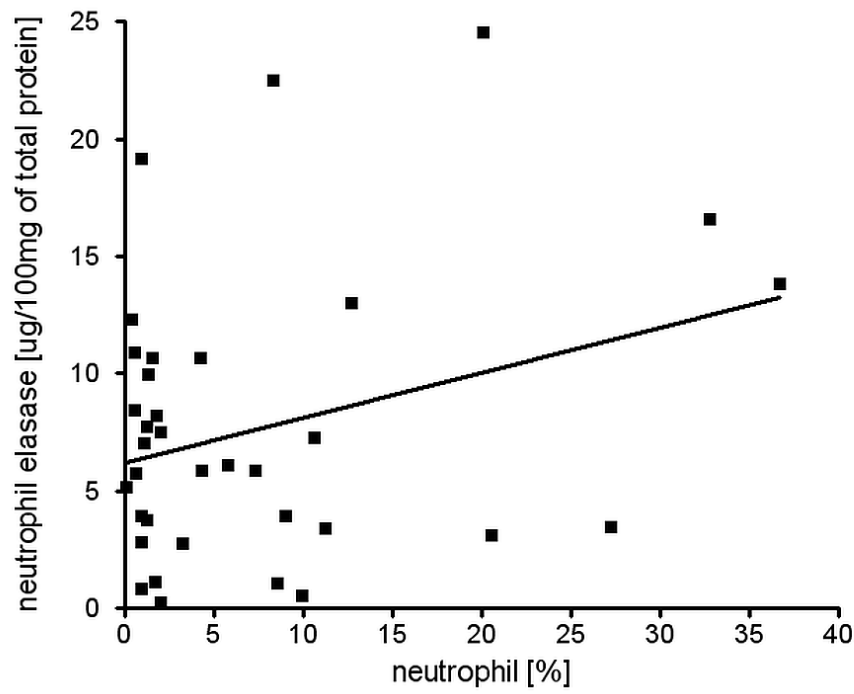

Figure 7 Spearman $r$ correlation between percentage of neutrophils in studied pleural effusions and concentration of neutrophil elastase (NE) measured against to the $100 \mathrm{mg} /$ of the total protein concentration present in the studied pleural effusions 
elastase increases the activity of the PI3K kinase, which enhances the interaction between PDGF and its receptor (PDGFR). The result of this process is an increase in the proliferation and survival of cancer cells [30]. AS Ho et al. highlighted the role of elastase as a diagnostic marker of the colon cancer. They showed that the concentration of NE in the serum of patients with colon cancer was significantly higher than in the healthy control [31]. We have found a comparable concentration of neutrophil elastase in pleural effusions, regardless of their etiology. However, Yamashita et al. [32] showed a concentration of NE in patients with lung cancer with the most advanced form of the disease, i.e. in T4, at a level similar to that observed by us. The observed high concentration of $\mathrm{NE}$ indicates that regardless of origin, pleural effusion is an environment with a strong inflammatory character. The proteolytic properties of elastase and the broad substrate spectrum of this enzyme may cause degradation of the structure of pleura membrane and contribute both to the recruitment of immune cells to the inside of the pleural cavity, also to easier penetration of metastatic cells migrating from the place of the primary tumor. The Spearman's rank correlation test did not show statistical significance between the mean concentration of NE in the examined pleural effusion and the percentage of neutrophils in this environment. However, the observed trend indicated a directly proportional relationship between the studied factors (Fig. 7). A slightly lower concentration of NE in malignant-derived pleural effusions can be associated with the immunosuppressive nature of this environment that may limit the secretory function of neutrophils. This inhibition may result in less efficient secretion of NE in malignant effusions than in non-malignant. It should be taken into account the multitude and variety of factors present in the pleural effusions, which are not fully known. Discovery other elements of the network of connections and dependencies between components of this environment may contribute to the identification of new targets for the cancer therapy.

\section{Conclusions}

Our studies demonstrate the functional plasticity of neutrophils and their variability depending on the factors present in the microenvironment in which they are found. Therefore, these cells are considered to be one of the targets of anticancer therapy, as elements susceptible to immunomodulation. Neutrophils immunomodulation gives the possibility of the creation of new therapeutic procedures based on attempts to restore the primary anticancer properties of these cells, depending on the advance of functional and immunophenotypic changes. However, in the absence of the possibility of immunomodulation, the total depletion of this cell population. This approach may be a good step towards personalized therapy for cancer.

\section{Acknowledgements}

The research described in this article was supported by grants from the Polish Ministry of Science and Higher Education No. N N402 684740, and Student Scientific Society of the University of Medical Sciences in Poznan No. 502-05-01127188-50746

\section{Author details}

Mariusz Kaczmarek, Ph.D., Department of Immunology, Chair of Clinical Immunology, Poznan University of Medical Sciences 5D Rokietnicka Str., 60-806 Poznan, Poland, phone (+48) 618547180, fax (+48) 618547173, e-mail: markacz@ump.edu.pl

\section{Conflict of interest statement}

We declare no conflict of interest

\section{References}

1. Karkhanis VS, Joshi JM. Pleural effusion: diagnosis, treatment, and management. Open Access Emerg Med. 2012;4:31-52; DOI:10.2147/OAEM. S29942.

2. Ried M, Hofmann HS. The treatment of pleural carcinosis with malignant pleural effusion. Dtsch Arztebl Int. 2013;110(18):313-18; DOI:10.3238/arztebl.2013.0313.

3. Noppen M, De Waele M, Li R, Gucht KV, D'Haese J, Gerlo E, Vincken W. Volume and cellular content of normal pleural fluid in humans examined by pleural lavage. Am J Respir Crit Care Med. 2000;162(3Pt1):1023-6; DOI:10.1164/ajrccm.162.3.9910050.

4. Light RW, Erozan YS, Ball CW. Cells in the pleural fluid: their value in differential diagnosis. Arch Intern Med. 1973;132(6):854-60; DOI:10.1001/ archinte.1973.03650120060011.

5. Bruehl RE, Moore KL, Lorant DE, Borregaard N, Zimmerman GA, McEver RP, Bainton DF. Leukocyte activation induces surface redistribution of P-selectin glycoprotein ligand-1. J Leukoc Biol. 1997;61(4):489-99; DOI:10.1002/jlb.61.4.489

6. Abram CL, Lowell CA. The ins and outs of leukocyte integrin signaling. Annu Rev Immunol. 2009;27:339-62; DOI:10.1146/annurev. immunol.021908.132554.

7. Etzioni A. Adhesion Molecules-Their Role in Health and Disease. Pediatr Res. 1996;39(2):191-8; DOI: 10.1203/00006450-199604001-01156.

8. Kolaczkowska E, Kubes P. Neutrophil recruitment and function in health and inflammation. Nat Rev Immunol. 2013;13(3):159-75; DOI:10.1038/ nri3399.

9. Repp R, Valerius T, Sendler A, Gramatzki M, Iro H, Kalden JR, Platzer E. Neutrophils express the high affinity receptor for IgG (Fc RI, CD64) after in vivo application of recombinant human granulocyte colony-stimulating factor. Blood. 1991;78(4):885-9.

10. Lukácsi S, Nagy-Baló Z, Erdei A, Sándor N, Bajtay Z. The role of CR3 (CD11b/CD18) and CR4 (CD11c/CD18) in complement-mediated phagocytosis and podosome formation by human phagocytes. Immunol Lett. 2017;189:64-72; DOI:10.1016/j.imlet.2017.05.014.

11. Robinson JM. Reactive oxygen species in phagocytic leukocyte. Histochem Cell Biol. 2008;130(2):281-97; DOI:10.1007/s00418-008-0461-4

12. Weinrauch Y, Drujan D, Shapiro SD , Weiss J, Zychlinsky A. Neutrophil elastase targets virulence factors of enterobacteria. Nature. 2002;417(6884):91-4; DOI:10.1038/417091a.

13. Janoff A, Schere J. Mediators of inflammation in leukocyte lysosomes. IX. Elastinolytic activity in granules of human polymorphonuclear leukocytes. J Exp Med. 1968;128(5):1137-55; DOI:10.1084/jem.128.5.1137.

14. McDonald JA, Kelly DG. Degradation of fibronectin by human leukocyte elastase: release of biologically active fragments. J Biol Chem. 1980;255(18):8848-58.

15. Mainardi C, Dixit S, Kang A. Degradation of type IV collagen (basement membrane) by a proteinase isolated from human polymorphonuclear leukocyte granules. J Biol Chem. 1980;255(11):5435-41.

16. Papayannopoulos V, Metzler KD, Hakkim A, Zychlinsky A. Neutrophil elastase and myeloperoxidase regulate the formation of neutrophil extracellular traps. J Cell Biol. 2010;191(3):677-91; DOI:10.1083/ jcb.201006052

17. Sarraf KM, Belcher E, Raevsky E, Nicholson AG, Goldstraw P, Lim E. Neutrophil/lymphocyte ratio and its association with survival after complete resection in non-small cell lung cancer. J Thorac Cardiovasc Surg. 2009;137(2):425-8; DOI:10.1016/j.jtcvs.2008.05.046.

18. Wu G, Yao Y, Bai C, Zeng J, Shi D, Gu X, Shi X, Song Y. Combination of platelet to lymphocyte ratio and neutrophil to lymphocyte ratio is a usefu prognostic factor in advanced non-small cell lung cancer patients. Thorac Cancer. 2015;6(3):275-87; DOI:10.1111/1759-7714.12178.

19. Halazun KJ, Hardy MA, Rana AA, Woodland DC, Luyten EJ, Mahadev S, Witkowski P, Siegel AB, Brown RS Jr, Emond JC. Negative impact of neu- 
trophil-lymphocyte ratio on outcome after liver transplantation for hepatocellular carcinoma. Ann Surg. 2009;250(1):141-51; DOI:10.1097/ SLA.0b013e3181a77e59.

20. He JR, Shen GP, Ren ZF, Qin H, Cui C, Zhang Y, Zeng YX, Jia WH. Pretreatment levels of peripheral neutrophils and lymphocytes as independent prognostic factors in patients with nasopharyngeal carcinoma. Head Neck. 2012;34(12):1769-76; DOI:10.1002/hed.22008.

21. Schmidt H, Bastjolt L, Geersten P, Christensen IJ, Larsen S, Gehl J, von der Maase H. Elevated neutrophils and monocyte counts in peripheral blood are associated with poor survival in patients with metastatic melanoma: a prognostic model. Br J Cancer. 2005;93(3):273-8; DOI:10.1038/ sj.bjc.6602702

22. Teramukai S, Kitano T, Kishida Y, Kawahara M, Kubota K, Komuta K, Minato K, Mio T, Fujita Y, Yonei T, Nakano K, Tsuboi M, Shibata K, Furuse K, Fukushima M. Pretreatment neutrophil count as an independent prognostic factor in advanced non-small-cell lung cancer: an analysis of Japan Multinational Trial Organisation LC00-03. Eur J Cancer. 2009;45(11):1950-8; DOI:10.1016/j.ejca.2009.01.023.

23. Trellakis S, Bruderek K, Dumitru CA, Gholaman H, Gu X, Bankfalvi A, Scherag A, Hütte J, Dominas N, Lehnerdt GF, Hoffmann TK, Lang S, Brandau S. Polymorphonuclear granulocytes in human head and neck cancer: enhanced inflammatory activity, modulation by cancer cells and expansion in advanced disease. Int J Cancer. 2011;129(9):2183-93; DOI:10.1002/ijc.25892.

24. McCourt M, Wang JH, Sookhai S, Redmond HP. Proinflammatory mediators stimulate neutrophil-directed angiogenesis. Arch Surg. 1999;134(12):1325-31; D0I:10.1001/archsurg.134.12.1325.

25. Coussens LM, Tinkle CL, Hanahan D, Werb Z. MMP-9 supplied by bone marrow-derived cells contributes to skin carcinogenesis. Cell. 2000;103(3):481-90; DOI:10.1016/S0092-8674(00)00139-2.

26. Kaczmarek M, Sikora J. Macrophages in malignant pleural effusions alternatively activated tumor associated macrophages. Contemp Oncol (Pozn). 2012;16(4):279-84; DOI:10.5114/wo.2012.30054.

27. Spicer JD, McDonald B, Cools-Lartigue JJ, Chow SC, Giannias B, Kubes P, Ferri LE. Neutrophils Promote Liver Metastasis via Mac-1-Mediated Interactions with Circulating Tumor Cells. Cancer Res. 2012;72(16):391927; DOI:10.1158/0008-5472.CAN-11-2393.

28. Ostrand-Rosenberg S, Sinha P. Myeloid-derived suppressor cells: linking inflammation and cancer. J Immunol. 2009;182(8):4499-506; DOI:10.4049/jimmunol.0802740.

29. Hurt B, Schulick R, Edil B, El Kasmi KC, Barnett C Jr. Cancer-promoting mechanisms of tumor-associated neutrophils. Am J Surg. 2017;214(5):938-944; DOI:10.1016/j.amjsurg.2017.08.003

30. Houghton AM. The paradox of tumor associated neutrophils. Fueling growth tumor with cytotoxic substances. Cell Cycle. 2010;9(9):1732-7 DOI:10.4161/cc.9.9.11297

31. Ho AS, Chen CH, Cheng CC, Wang CC, Lin HC, Luo TY, Lien GS, Chang J. Neutrophil elastase as a diagnostic marker and therapeutic target in colorectal cancers. Oncotarget. 2014;5(2):473-80; DOI:10.18632/ oncotarget.1631.

32. Yamashita J, Ogawa M, Abe M, Hayashi N, Kurusu Y, Kawahara K, Shirakusa T. Tumor neutrophil elastase is closely associated with the direct extension of non-small cell lung cancer into the aorta. Chest. 1997;111(4):885-90; DOI:10.1378/chest.111.4.885. 